\title{
LION: laser interferometer on the moon
}

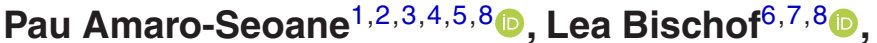 \\ Jonathan J. Carter ${ }^{6,7,8, *}\left(\mathbb{D}\right.$, Marie-Sophie Hartig ${ }^{6,7,8}$ (1) and \\ Dennis Wilken ${ }^{6,7,8}$ \\ 1 Universitat Politècnica de València, IGIC, Spain \\ 2 DESY, Zeuthen, Germany \\ ${ }^{3}$ Kavli Institute for Astronomy and Astrophysics, People's Republic of China \\ ${ }^{4}$ Institute of Applied Mathematics, Academy of Mathematics and Systems Science, \\ People's Republic of China \\ 5 Zentrum für Astronomie und Astrophysik, TU Berlin, Germany \\ ${ }^{6}$ Albert Einstein Institute, Max Planck Institute for Gravitational Physics, Hannover \\ 30167, Germany \\ 7 Albert-Einstein-Institut, Leibniz Universität Hannover, Hannover 30167, Germany \\ E-mail: Jonathan.Carter@aei.mpg.de
}

Received 18 December 2020, revised 30 March 2021

Accepted for publication 1 April 2021

Published 24 May 2021

\begin{abstract}
Gravitational wave astronomy has now left its infancy and has become an important tool for probing the most violent phenomena in our Universe. The LIGO/Virgo-KAGRA collaboration operates ground based detectors which cover the frequency band from $10 \mathrm{~Hz}$ to the $\mathrm{kHz}$ regime. Meanwhile, the pulsar timing array and the soon to launch LISA mission will cover frequencies below $0.1 \mathrm{~Hz}$, leaving a gap in detectable gravitational wave frequencies. Here we show how a laser interferometer on the moon (LION) gravitational wave detector would be sensitive to frequencies from sub $\mathrm{Hz}$ to $\mathrm{kHz}$. We find that the sensitivity curve is such that LION can measure compact binaries with masses between 10 and $100 M_{\odot}$ at cosmological distances, with redshifts as high as $z=100$ and beyond, depending on the spin and the mass ratio of the binaries. LION can detect binaries of compact objects with higher-masses, with very large signal-to-noise ratios (SNRs), help us to understand how supermassive
\end{abstract}

\footnotetext{
${ }^{8}$ All authors contributed equally to this work.

*Author to whom any correspondence should be addressed.
}

Original content from this work may be used under the terms of the Creative Commons Attribution 4.0 licence. Any further distribution of this work must maintain attribution to the author(s) and the title of the work, journal citation and DOI. 
black holes got their colossal masses on the cosmological landscape, and it can observe in detail intermediate-mass ratio inspirals at distances as large as at least $100 \mathrm{Gpc}$. Compact binaries that never reach the LIGO/Virgo sensitivity band can spend significant amounts of time in the LION band, while sources present in the LISA band can be picked up by the detector and observed until their final merger. Since LION covers the deci-Hertz regime with such large SNRs, it truly achieves the dream of multi messenger astronomy.

Keywords: gravitational wave detector, lunar, detector concepts, deci-Hertz

(Some figures may appear in colour only in the online journal)

\section{Introduction}

The LIGO-Virgo-KAGRA (LVK) collaboration [1-3] now forms a network of gravitational wave observatories covering the Earth. Third generation ground based detectors such as the Einstein telescope (ET) [4] and cosmic explorer (CE) [5, 6] are currently in the planning stage of development. These detectors are planned to push the lower frequency bound of Earth based gravitational wave detectors to their fundamental limits.

Newtonian noise, or gravitational gradient noise, forms a lower frequency bound of Earth based detection at about $1 \mathrm{~Hz}$ [7]. Meanwhile, with a proposed launch of 2034, the ESA's LISA mission [8] will cover the $\mu \mathrm{Hz}$ to sub deci-Hz regime of the gravitational wave spectrum. The upper sensitivity bound of space based detectors is set by the interferometer arm length $[9,10]$.

These barriers create a gap of measurable frequencies which requires a different style of mission to fill. In this gap many interesting stellar phenomena go unrecorded and their immeasurable impact on the field of gravitational wave astronomy is lost [11]. Therefore, there is great interest in any gravitational wave detector capable of bridging this gap. An ambitious mission proposed by the Japanese gravitational community called DECIGO is in the planning stage of development [12]. DECIGO aims to cover the deci-Hertz band; however, it is a new style of detector, which requires many technological innovations before it can launch [13, 14]. This mission, and others [15], are still a long way from realisation, so in order to cover these frequencies in the operational time of third generation detectors, other avenues of low frequency detection must be explored. In this work we show that a lunar gravitational wave observatory would be able to observe frequencies down to $0.7 \mathrm{~Hz}$ while simultaneously overlapping with the sensitive frequency range of Earth based detectors.

During the 90s Wilson and La Fave published two papers on the idea of lunar interferometers, but as the field was still in its infancy, a lot of benefits and challenges went overlooked $[16,17]$. A few other groups have suggested using seismometer arrays on both the Moon and other extraterrestrial solar bodies $[18,19]$. Coughlin and Harms managed to put limits on the energy contained within the stochastic background across the frequency band $0.1-1 \mathrm{~Hz}$ using seismometers on the Moon from the Apollo missions as a detector. A recent proposal has now been submitted to launch many high sensitivity seismometers to the surface of the Moon to push this detection method to its limits [20]. If launched, these seismometers would enable a better understanding of the Moon's seismic activity and so the true limits of seismic noise as discussed later in this paper. Aside from that, a paper has been submitted to arxiv recently discussing the concept of a lunar gravitational wave detector [21]. They focus largely on the astrophysical sources in their sensitivity band. Although their optimistic estimate of performance was excellent at low frequency, it relied upon the use of a freely floating test mass, a technology that has only been proposed and has no further development so far [22]. The 
Table 1. Overview of the key parameters of LION. The masses and suspension length are listed from test mass to top mass. The model is based on the gravitational wave interferometer noise calculator (gwinc) CE 2 from October 2020. The full model and adapted gwinc software is provided as supplementary material. The full detector configuration files can be found at https://github.com/Jonjocarts/LION-Public.

\begin{tabular}{lc}
\hline Parameter & Value \\
\hline Laser power & $50 \mathrm{~W}$ \\
Wavelength & $2000 \mathrm{~nm}$ \\
Arm length & $40 \mathrm{~km}$ \\
Seismic & Earth surface $/ 1000$ \\
Test mass & $1267 / 1267 / 698 / 707 \mathrm{~kg}$ \\
ITM radius of curvature & $34 \mathrm{~km}$ \\
ETM radius of curvature & $36 \mathrm{~km}$ \\
Suspension length & $3.54 / 2.05 / 1.66 / 2.50 \mathrm{~m}$ \\
Temperature & $70 \mathrm{~K}$ \\
Signal recycling cavity length & $55 \mathrm{~m}$ \\
Squeezing & $15 \mathrm{~dB}$ \\
Initial squeezing level & $8.94 \mathrm{~km}$ \\
Filter cavity length & $-2.3 \mathrm{~Hz}$ \\
Filter cavity detuning & \\
Mirror transmittance & $5 \mathrm{ppm}$ \\
End test mass & $1.2 \%$ \\
Input test mass & $3 \%$ \\
Power recycling mirror & $2 \%$ \\
Signal recycling mirror & $0.17 \%$ \\
Filter cavity input mirror & $5 \mathrm{ppm}$ \\
Filter cavity end mirror & \\
\hline
\end{tabular}

conservative estimate they make is similar to the one we reach here, without focusing on the technical details needed to reach that. We also show significant differences in detector design. Still, it is very promising that two independent approaches can reach similar noise estimates.

In the following we discuss how a ground based long arm interferometer with Fabry-Pérot cavities could be constructed for use as a gravitational wave detector on the surface of the Moon. We will discuss the merits and potential design style of such a lunar observatory. We address this by constructing a feasible noise budget for a laser observatory based upon current gravitational wave detectors. We benefit from the lunar surface's seismically quiet environment [23], and its lack of atmosphere. Thus the limit on low frequency detection set by the Newtonian noise can be avoided. By means of this noise budget, we show that the sensitivity gap between the high frequency ground based detectors and low frequency space based detectors can be partially bridged. Furthermore, a detector built on the Moon with a sensitivity band also overlapping that of the Earth based network increases the maximal distance between the detectors by a factor of 30, improving the source localisation ability significantly.

Several stellar phenomena are expected in the frequency band exclusively covered by our proposed lunar detector and we discuss the impact their observations will have. A low frequency detector allows the tracking of merging binaries for much longer times [24], detecting binaries at much greater redshift [25], detecting heavier binaries [26], allows for better sky localisation [27], and detecting new types of astrophysical sources [28, 29]. Long standing questions about the origins of many sources could uniquely be resolved with a LION detector [30]. Furthermore, sub-Hertz detection allows for the probing of gravity in the strong field 


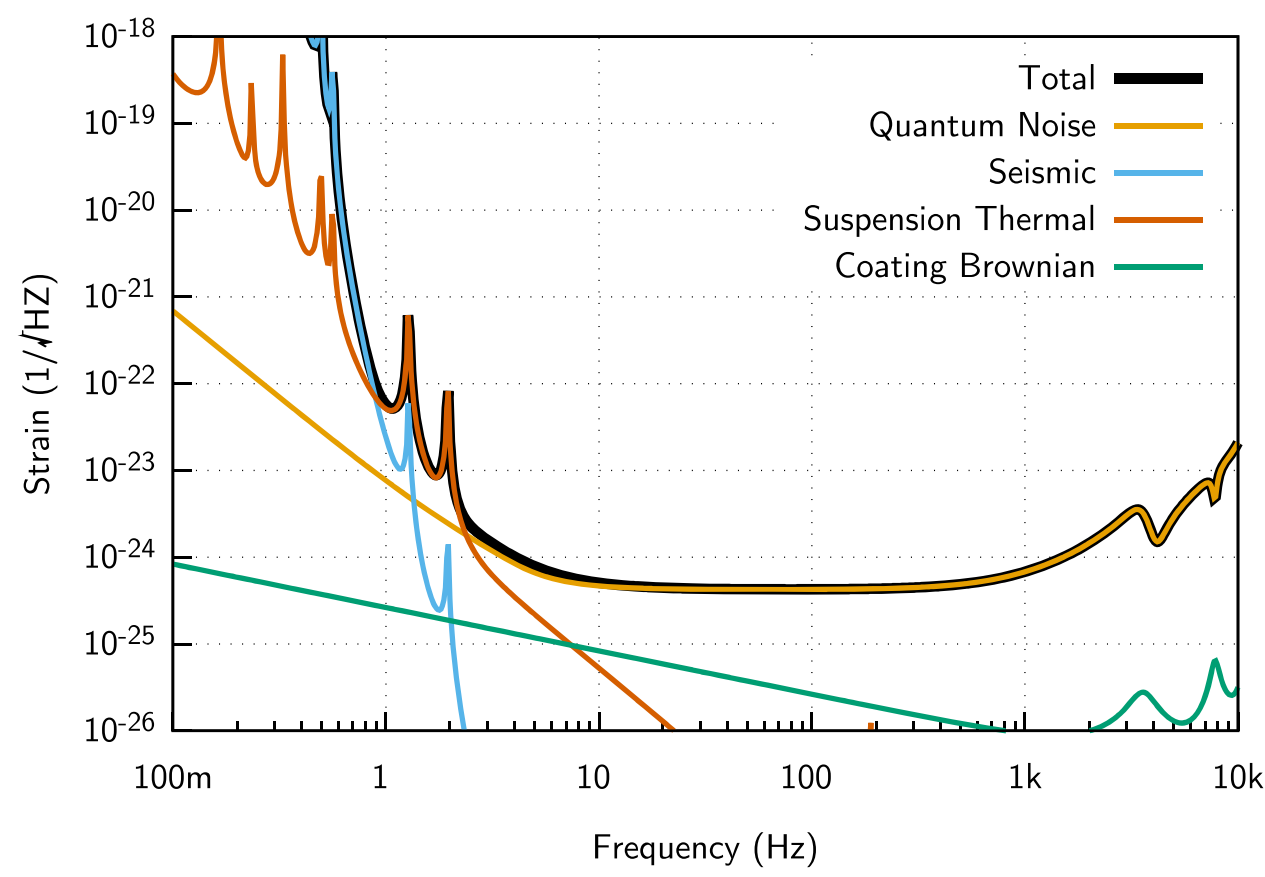

Figure 1. Noise budget for a gravitational wave detector operated on the Moon with an arm length of $40 \mathrm{~km}$ and optics with increased masses at a temperature of $70 \mathrm{~K}$. The parameters used to create this are listed in table 1 . The detector is quantum noise limited above $2 \mathrm{~Hz}$ and limited by thermal noise below this until it hits the seismic cliff at $0.8 \mathrm{~Hz}$. The bandwidth of the detector is therefore approximately from $0.7 \mathrm{~Hz}$ to the kHz. Simulated with gwinc [38].

regime, testing general relativity to its limits [31-33]. Finding sources at very high redshifts leads directly to a better understanding of the early Universe, where our current understanding is limited by our detection capabilities.

With the renewed interest in lunar missions from several major global powers [34-37], now is the ideal time for the gravitational wave community to begin considering what use the Moon can be in aiding our understanding of the cosmos.

Section 2 starts with the discussion of technical details, including detector parameters, noise contributions and the location and infrastructure. This is followed in section 3 by a summarised noise budget. The science case in section 4 discusses the new possibilities that a lunar detector offers, while section 5 gives an estimate about the mission cost and timeline.

\section{Technical discussion}

The model for our suggested laser interferometer on the moon (LION) is based on the CE 2 proposal. The key parameters are listed in table 1. Our changes arise from the special conditions on the Moon and design choices made to optimise the frequency band of interest which we discuss below. This leads to the noise curves shown in figure 1 . The overall schematic is shown in figure 2 . 


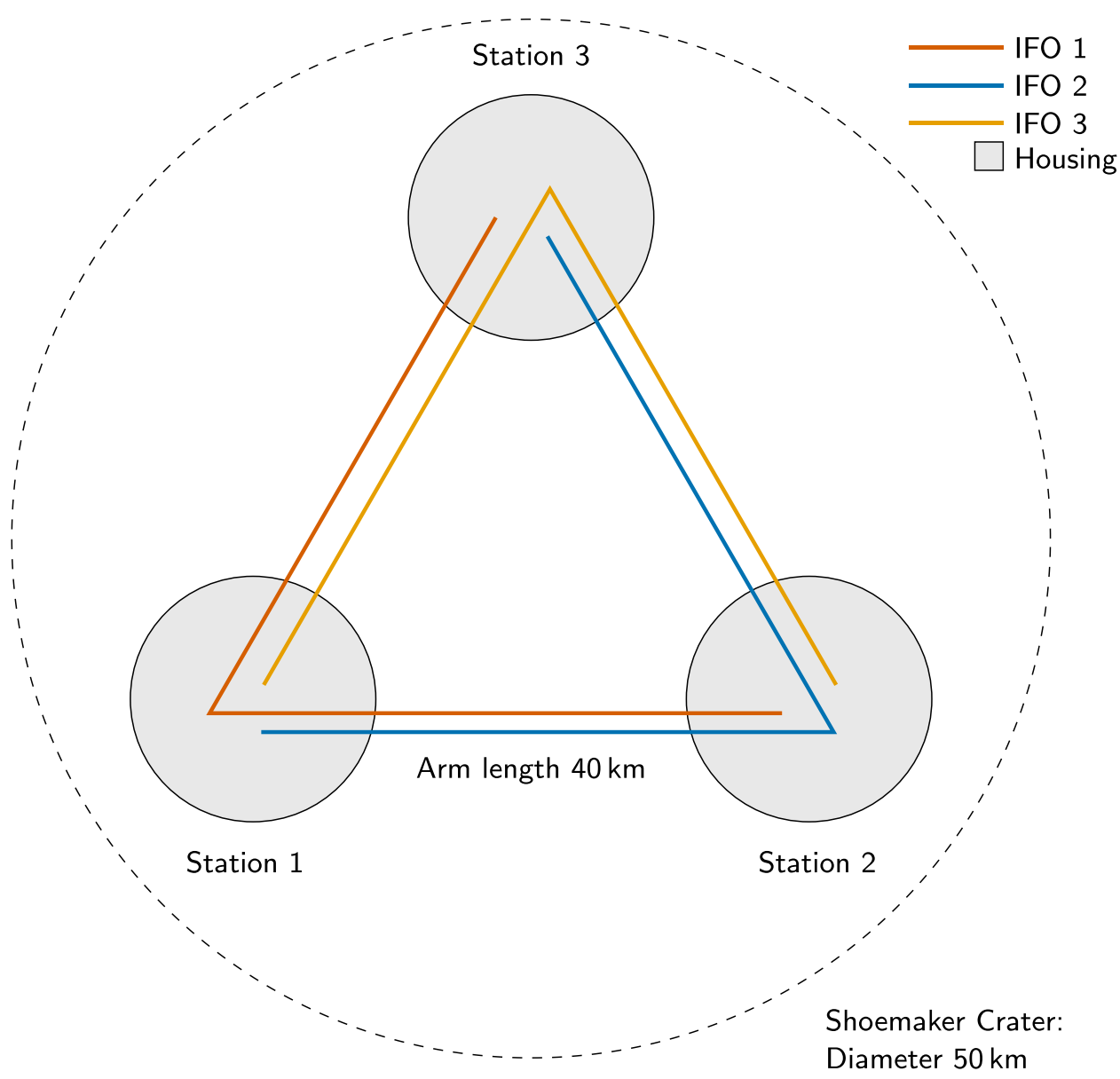

Figure 2. A schematic representation of the LION detector, showing the three interferometers and end test stations. The arms are all $40 \mathrm{~km}$ in length. The end stations must be protected from dust entering them and each contain isolation platforms and suspension systems for the core and auxiliary optics.

\subsection{Location}

The choice of the location for LION is predominately determined by the temperature map of the Moon. A crater near a lunar pole is considered by us to be a suitable position for several reasons; thermal stability, geometry, and shelter from solar radiation and dust [39-41]. A promising candidate site is the Shoemaker crater $\left(88.1^{\circ} \mathrm{S} 44.9^{\circ} \mathrm{E}\right)$ at the Moon's south pole. Hayne et al [39] investigated the measurement data of the lunar reconnaissance orbiter (LRO) mission launched in 2009 for three craters with about $50 \mathrm{~km}$ diameter. The shoemaker crater shows an average temperature of about $50 \mathrm{~K}$ with a relatively small variation of minimal/maximal temperature $20-95 \mathrm{~K}$ compared to other parts of the Moon. It has a diameter of $50 \mathrm{~km}$, introducing one of the limiting factors for the detectors: the arm length. The nearby Malapert mountain is a nearly perfect site to build solar panels and a communication equipment for data transfer [42]. Additionally, the surface appears to be nearly free of water frost, which 
could otherwise evaporate onto optics [39]. We assume the use of this crater for all future calculations.

\subsection{Infrastructure}

The vacuum on the surface of the Moon is three orders of magnitude better than that in the vacuum tubes in the aLIGO detectors [1,43], and so no infrastructure will be built to house a vacuum. Only the optic support stations are needed to isolate the test optics and allow for their precise alignment controls.

For the detection of gravitational waves, coincidence data from two independent simultaneous instruments is required. This is solved in the ET detector and LISA by using a triangular shape with three interferometers in the arms [4, 44, 45]. Therefore, we propose to build LION in a triangular shape with an arm length of $40 \mathrm{~km}$, limited by the dimensions of the crater. The three interferometers of LION will also allow for the detection of gravitational waves regardless of polarisation, as shown in figure 2 .

\subsection{Background seismic}

The seismic noise on the Moon arises from two main sources; meteorite impacts [46-49] and from the shallow seismic activity of the Moon [50]. Many of the sources of seismic activity on Earth do not exist on the Moon [51] such as moving cars and trains, winds, and the oceans tides. In particular, the oceans tides cause a large microseismic peak at $0.1-0.2 \mathrm{~Hz}$ which proves troublesome to all detectors seeking low frequency performance; a lunar site completely negates this [52]. Meanwhile, wind and ground conditions on the Earth tilts the detectors, polluting the measurements of inertial sensors with additional noise, which is directly injected into platform controls $[53,54]$. The absence, or at the very least substantial reduction, of tilt would allow for a much more aggressive active platform control scheme to reduce motion in the test masses [55]. The control noise is currently one of the reasons the aLIGO detectors do not reach their design sensitivity at low frequencies.

The only direct seismic measurements on the Moon's surface were performed during the Apollo missions $[23,46]$. They concluded that the background seismic level was significantly below the sensitivity of their measurement devices $\left(0.3 \mathrm{~nm} \mathrm{~Hz}^{-1 / 2}\right.$ at $\left.1 \mathrm{~Hz}\right)$, and thus lower than anything on Earth's surface. From this we can safely assume the seismic on the Moon to be smaller by a factor of 1000 than Earth's surface seismic motion. However, the Russian operated Luna 27 will be launched in 2024 [56] with a more sensitive seismometer [57], possibly measuring an even lower boundary.

\subsection{Newtonian noise}

Newtonian noise is caused by the movement of mass around the detector causing a change in the local gravitational field [51]. These largely come from two sources; the atmosphere and the ground. The lack of atmosphere on the Moon negates the former entirely. Reliable modeling of seismic Newtonian noise for the lunar surface is impossible without accurate measurements of the seismic activity and surface composition. On Earth the noise forms a noise cliff at approximately $1 \mathrm{~Hz}$. Since the Moon has lower activity [47] and has a lower density on its surface it is assumed that this will occur at a lower frequency. Newtonian noise is therefore not plotted in the noise budget. A full study of the gradient noise would still be essential as more knowledge of the Moon's activity is gained. 


\subsection{Suspension systems}

The limiting low frequency noise considerations are the residual of seismic activity and suspension thermal noise. Like in Earth bound detectors, a multistage seismic isolation system would be necessary. This will comprise of a preisolation stage and several suspended masses each acting as a pendulum $[58,59]$. In order to achieve the required seismic isolation, the resonance frequencies of the pendula must be tuned to lower frequency than CE. The gravity on the Moon's surface is $1.62 \mathrm{~m} \mathrm{~s}^{-2}$, approximately a sixth of the gravity on Earth. Although the lower gravity of the Moon offers lower natural frequency by a factor of 0.4 , longer suspensions will still be needed. Furthermore, to help combat thermal noise, heavier test masses must still be included. As the gravity is weaker, the masses can be a factor of 6 larger than CE without additional weight on the suspensions. Making such large test masses and coating them may prove difficult so we assume only a factor of 4 increase can be made. This leads to a test and penultimate mass of $1270 \mathrm{~kg}$ and another 2 stages with about $700 \mathrm{~kg}$ mass. Making the lengths of suspensions on average a factor of 3 longer allows for the whole system to fit in a $11 \mathrm{~m}$ structure while reducing the noise to a level suitable for sub $\mathrm{Hz}$ measurements. Suspension systems are a limiting factor for the low frequency performance of all detectors with fundamental problems of how much weight can be suspended and resonance frequency. The lower gravity on the Moon provides help on both of these fronts. How far this can be developed will form the lower boundary of measurable frequencies, but ultimately it is a matter of engineering.

\subsection{Thermal noise}

Thermal noise is a considerable problem for the low frequency response of any detector. The extremely low temperatures at the Moon's south pole are a good start for achieving the necessary thermal noise suppression. However, a low mechanical loss is also a necessity for a low thermal noise [60]. The bulk material of the test masses at both LIGO and Virgo are fused silica which, due to a mechanical loss peak, has relatively high thermal noise at cryogenic temperatures [61]. Research for alternative test mass materials is ongoing for use in the cryogenic interferometer of ET and other detectors [62-64].

The CE proposal uses silicon ribbons to suspend their test masses and research is ongoing to design the next generation of suspensions [65]. The properties of the ribbons quoted in the CE design is assumed when calculating the suspension thermal noise.

Likewise, significant progress is needed to reduce both mechanical and optical losses of the test mass coatings to reach CE's design sensitivity [6]. The progress of these development will be at the forefront of the efforts of next generation of gravitational wave detectors and their results will heavily influence the limits of a lunar interferometer. The LION design does not need to exceed the performance of CE in this regard so it is assumed that if CE can meet the design sensitivity, the design can be copied for LION. Promising results in this regards are already being achieved by two means of coating; one with alternate material layer deposition [66] and the other by the structuring of resonant waveguides to create a highly reflective surface $[67,68]$.

\subsection{Quantum noise}

Quantum noise is the predominant noise source at high frequencies for Earth bound interferometric gravitational wave detectors. While frequency independent squeezing is injected in current detectors [69-71] to reduce the shot noise, there are plans for a broadband noise reduction by using filter cavities [72] or EPR entanglement [73]. The proposed LION design uses $50 \mathrm{~W}$ laser power, $15 \mathrm{~dB}$ initial squeezing and filter cavities. Compared to the $\mathrm{CE}$ design the 
power was reduced by a factor of 5 to increase the sensitivity at low frequencies. The detector will be quantum noise limited above $2 \mathrm{~Hz}$.

\subsection{Seismic shocks and asteroid impacts}

Another cause of concern is asteroid impacts on the Moon. A large impact near the equipment could potentially lead both to saturated isolation equipment leading to damage to the controls as well as large mirror motion and loss of science mode. Reacquiring science mode is a difficult and time consuming process $[74,75]$, however, a similar problem is encountered on Earth with earthquakes. This problem is tackled at aLIGO with a combination of a specialised early warning system for incoming high activity seismic waves and alternative control schemes for high activity times [76]. A similar strategy could be copied for any lunar setup. Therefore, only a direct hit on the experiment would present a problem.

Based on the data of the still ongoing LRO, approximately 47000 new impact sites had been detected in the area studied ( $6 \%$ of the lunar surface) in a 1241 day period [77]. This leads to an estimate of approximately 5 impacts within the detector area (a triangle with $40 \mathrm{~km}$ long sides) in a year. It is foreseen that this will prove to be a regular nuisance, but should still enable long stretches of observation. It is also unlikely that the optics will be hit directly as they will take up a small fractional area of the detector, leading to an estimate of direct hits once in a thousand years. Smaller impacts will be significantly under-counted by their very nature, and so it is likely a asteroid shielding solution of the optics will be needed.

\subsection{Dust accumulation}

The Apollo missions showed that the Moon's surface is covered in dust, which is kicked up by both activity, such as asteroid impacts and moonquakes, and solar winds [78, 79] and then coats everything on the lunar surface. This dust presents a very real challenge to the LION mission as all current gravitational wave detectors operate in the highest level of clean room environment. Dust stuck to the mirrors would act as a loss channel leading to an increase in quantum noise [80]. The increase in noise corresponding to different optical losses is shown in figure 3. To be a useful contribution to a gravitational wave network, optical losses at the mirror or along the path must be maintained below $0.1 \%$. In addition, each dust particle will act as a point absorber on the mirror. The LIGO detectors have discovered that these point absorbers exist in their mirrors, which absorb more of the light until they are heated enough to damage the mirrors [81]. It is, therefore, imperative that the mirrors remain as immaculate as possible. Due again to the need to suppress thermal noise, the material suspending the optics must also remain protected from dust. Any dust which does accumulate will degrade the quality factor of the suspensions and so lead to worse performance.

Dust does present a problem for many experiments on the Moon and other bodies in the Solar System; hence, much research is ongoing to combat this [82-91]. For LION, two dust reduction methods are promising, yet. Firstly, we mitigate the dust to stuck on our instruments by building everything in a closed housing, only accessible for the beam by a hole in direction of the beam path. The diameter of the hole must be at least three times the diameter of the beam to avoid a reduction of power circulating in the cavity. For a beam diameter of $360 \mathrm{~mm}$ this would give a hole diameter of about a meter. To prevent the dust from entering the housing via this hole, we consider adding a tunnel of a few meters length in front of the hole. This solution would be the simplest mechanically and cost wise. It may be the case that, after testing, is it found that further measures are required. To this end the second proposed mitigation technique for LION is an electrostatic dust shield at the instrument housings, which is currently considered to be the state of the art for cleaning optical elements [92]. Here, electrodes printed on a glass substrate 


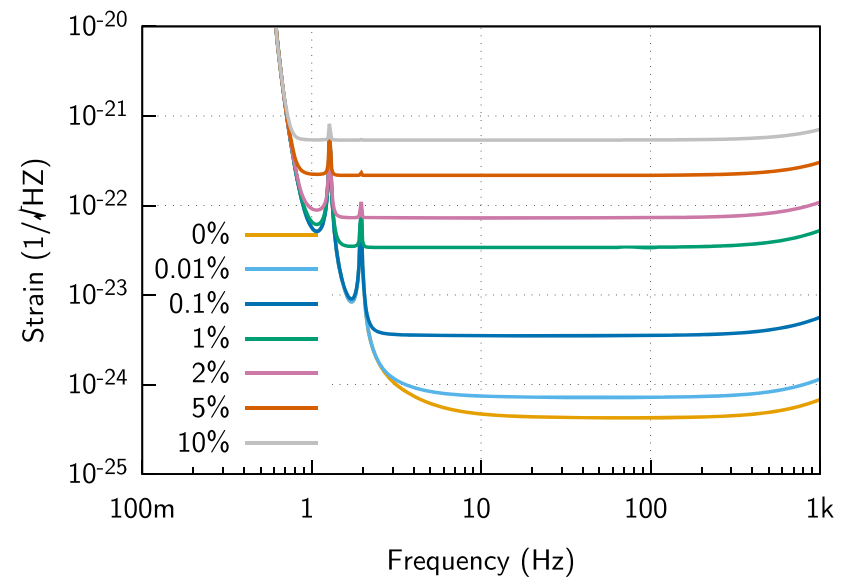

Figure 3. The effect of additional optical losses from dust on the mirror on the noise of the detector. The losses refer to optical power loss, per one travel of the cavity. Above a loss of $0.1 \%$ the detector makes few contributions to any proposed network and so a means of cleaning to this amount must be found. Losses highly influence, the frequency response of the interferometer and thus the filter cavity design. Therefore, we have assumed an optimised squeezing angel for this calculations, which artificially improves the low-frequency behavior of the $0 \%$ and $0.1 \%$ curves.
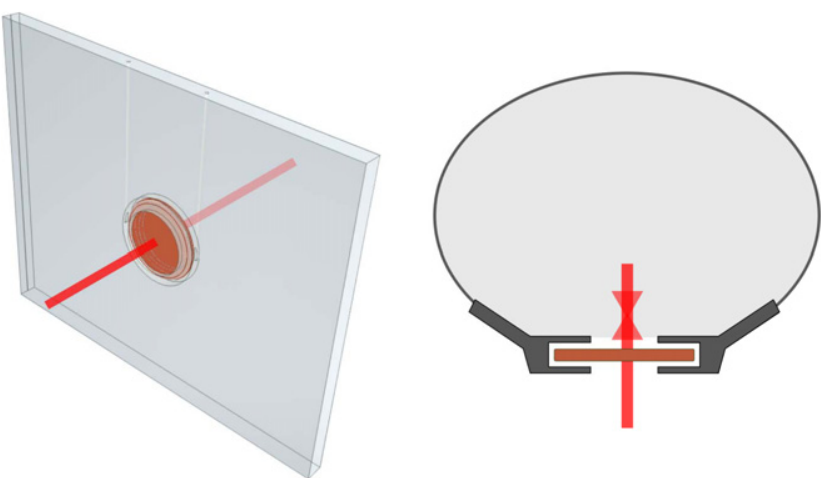

Figure 4. Schematic three dimensional view (left) and top view (right) of the dust protection method at the end stations, where an optic at the stations functions as an electrostatic dust shield. Since the optic has to be suspended, the station cannot be enclosed completely. Therefore, the wall is constructed around the window with tubes for the suspension, as shown in the left picture. The top view on the right side shows the resulting, very small space, that remains between the housing and the optic. The optical system including the test masses are placed behind this window and are not shown in this sketch.

move the electrostatic field toward the outer part of the optic. When utilised, the electrostatic wave removes both the uncharged particles as well as dust particles charged by the irradiation of solar winds and cosmic rays. Kawamoto et al have shown that electrostatic cleaning can limit the transparency loss to below $9 \%$ in experiments $[87,88]$. This will likely be improved by the orientation of the optics in LION which are placed orthogonal to the lunar surface. Hence no gravitational forces will hold the dust particles at their surface. Further research on 


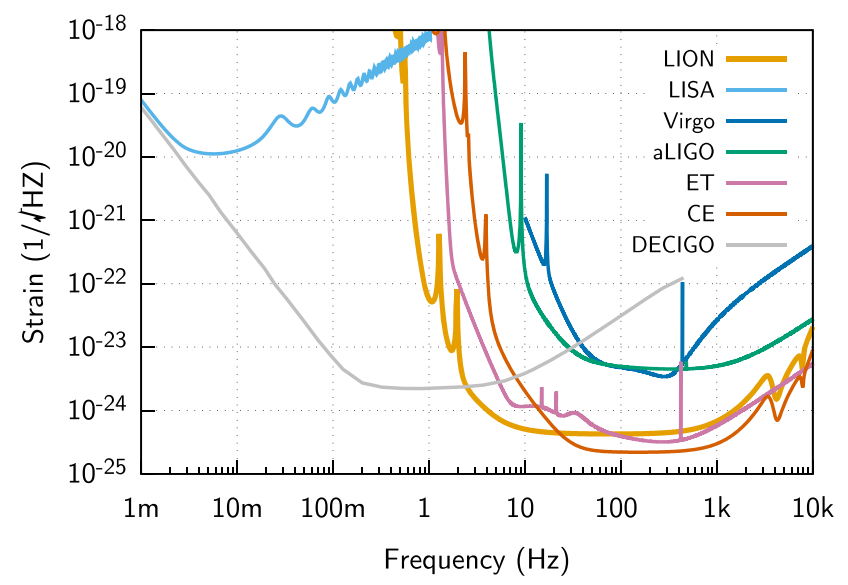

Figure 5. Comparison of the design sensitive of current and future gravitational wave detectors. Our proposed detector, LION, could extend the range of ground based detectors reducing the gap of measurable frequencies between Earth based and space-borne missions. LION has the highest sensitivity in the detection band from below $30 \mathrm{~Hz}$ and is the only detector able to with sufficient sensitivity for detection between $0.7 \mathrm{~Hz}$ and $2 \mathrm{~Hz}$.

this topic will show if this technique can be refined to a sufficient level. If an electrostatic dust shield were to be implemented, it would have to be suspended to isolate it from environmental conditions. A schematic view of this is shown in figure 4.

\subsection{Additional challenges}

A mission of this style will require several large payloads to be transported to the Moon and positioned carefully, requiring assembly either by hand or robot. The feasibility of these strongly depends directly on the lunar missions in the coming decade. However, technology is reaching the stage where commercial missions to the Moon are becoming viable, a promising sign of things to come [93].

The total mass of the suspended optics is $4000 \mathrm{~kg}$, and the weight of a preisolation stage used at LIGO is $2000 \mathrm{~kg}$ [52]. This would leave over $10000 \mathrm{~kg}$ as remaining load for both the structure and auxiliary optics, if it was to be carried by the highest capacity rocket today. This would require at least 3 launches for total transport.

Gravitational wave detectors produce a high amount of data due to the number of sensors monitoring the numerous degrees of freedom. Furthermore, many systems will have to be operated from ground stations. A high bandwidth connection will be required to the site. Currently a record of 622 Megabits/s data transfer rate has been achieved by the lunar laser communication demonstration in orbit of the Moon [94]. With an encoding of 64 bits per data point, we would be able to read out about 100 channels at a $100 \mathrm{kHz}$ sample rate. This $100 \mathrm{kHz}$ rate is an upper estimate of the data rate needed. While this may not encompass all of the data producing instruments, it should be sufficient for instrument readout of the main channel and diagnostics of auxiliary channels of interest to above the upper limits of LION's sensitive band.

With no need for a vacuum pump, and only small amounts of power needed for alignment controls and isolation, the main power consumption concerns are the telemetry, the high laser power, beam preparation and readout electronics, and the auxiliary sensors. In space missions the power supply is usually covered by a combination of advanced solar panels that reach an 

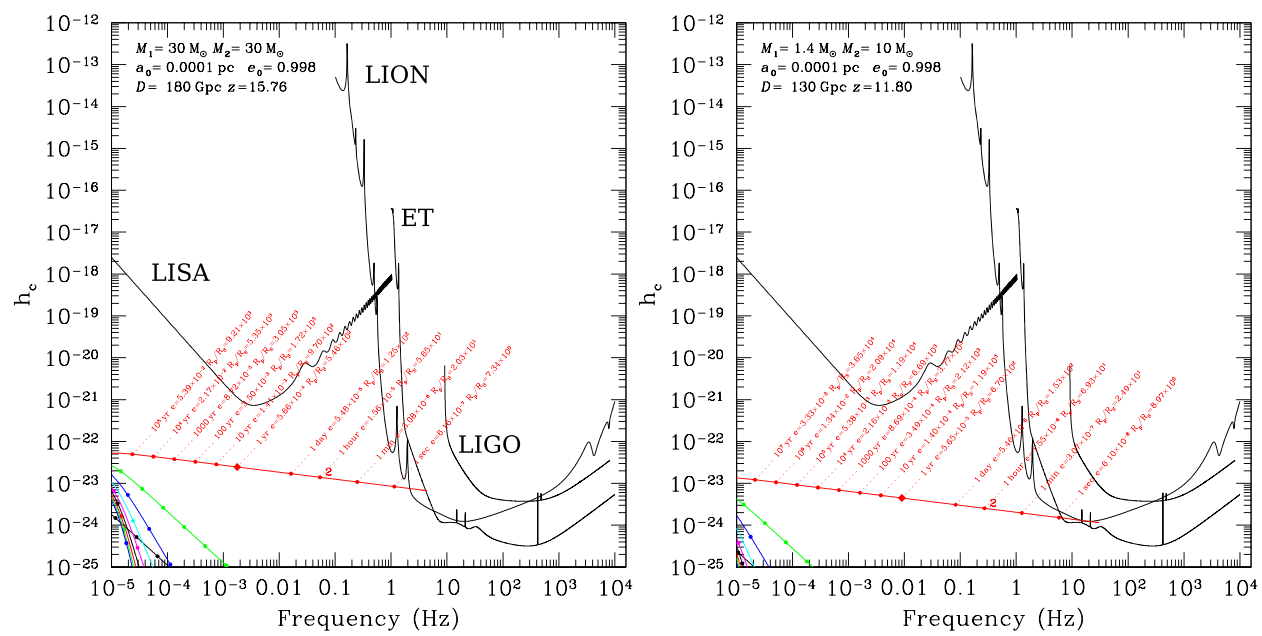

Figure 6. Evolution in frequency of the first harmonics (displayed in different colors) of two compact binaries in the approximation of Keplerian ellipses of [99]. We depict the characteristic, dimensionless amplitude as a function of the frequency in $\mathrm{Hz}$. The initial dynamical parameters corresponding to the each source is summarised at the top, left corner of each panel (masses, initial semi-major axis and eccentricity and distance to the source $D$ ). From the left (lower frequencies) to the right (higher frequencies), we show the sensitivity curve of LISA [8], then LION, starting at about $1 \mathrm{~Hz}$, the ET [100], and then LIGO. The upper, red harmonic corresponds to $n=2$ in equation (2), which means that the source has circularised. We also show different moments in the evolution of the harmonics, and add the information relative to the source at that moment; in particular the remaining time until merger, the eccentricity and the periapsis distance $R_{\mathrm{p}}$ in Schwarzschild radii $R_{\mathrm{S}}$. The left panel shows a binary of masses $M_{1}=M_{2}=30 M_{\odot}$ at a distance of $180 \mathrm{Gpc}$, which corresponds to a redshift of $z=15.76$. This is orders of magnitude distance further away than what LISA can hope to observe. The right panel shows the inspiral of a compact object of mass $1.4 M_{\odot}$ (e.g. a neutron star) onto a stellar-mass black hole of mass $10 M_{\odot}$ at a distance of $130 \mathrm{Gpc}(z=11.80)$. Even at this cosmological distance, the source spends one second in the LION domain. We note that no other detector is in the position of resolving these sources.

efficiency of 30\% and Li-Ion batteries. While at the equatorial and mid-latitude regions of the Moon the long lunar nights set limitations for solar power supply, we take advantage of locating the interferometer in polar regions. As shown by [42] we receive up to $93 \%$ of full or partial sunlight when placing the solar panels on mountains, for example the Malapert mountain, close to the chosen site. For $1.4 \mathrm{~kW} \mathrm{~m}^{-2}$ of power provided by the Sun and a pessimistic factor of 0.5 for the partial sunlight, we gain $0.2 \mathrm{~kW} \mathrm{~m}^{-2}$ of solar power, making the power of the system very manageable with a solar panel array of a few tens of square meters.

\section{Noise budget}

We used the gwinc [38] software to estimate the noise budget of our proposed LION design shown in figure 1. Cosmic Explorer (CE2 model, October 2020 [6]) was used as a base model, but with the changes discussed in the previous section and listed in table 1 to match the design presented in this paper. The model is currently limited at low frequencies by the properties of the suspension system. Figure 5 shows the LION noise budget estimate and compares it with the performance of other current and proposed ground based detectors and the space 

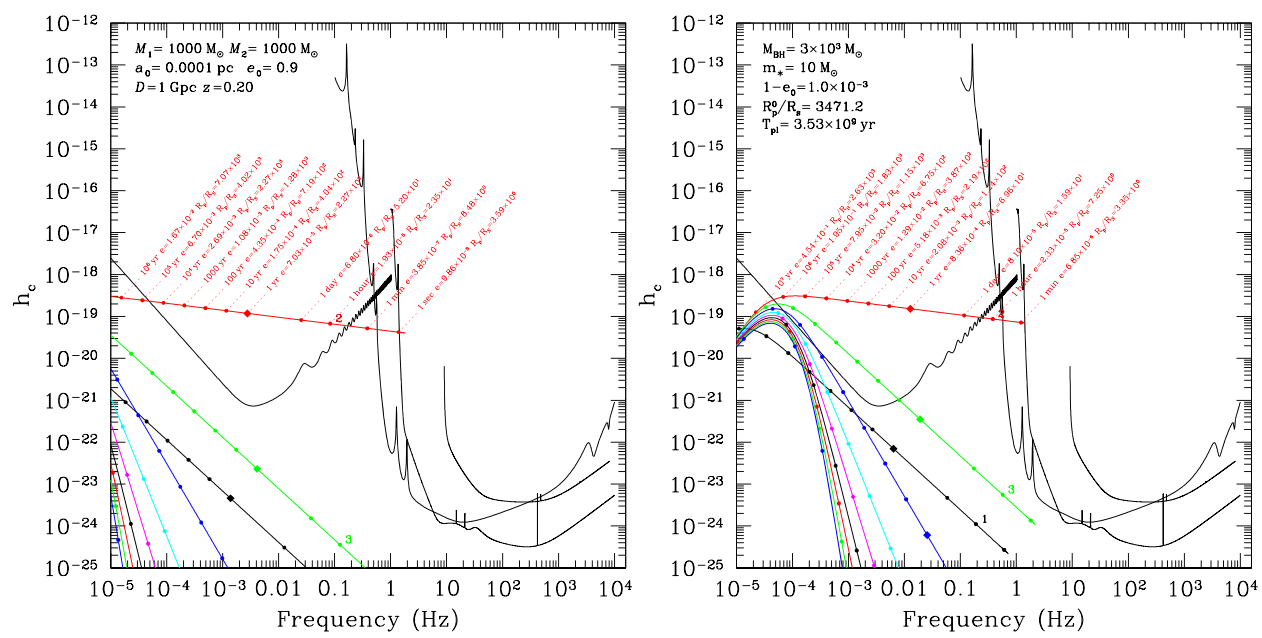

Figure 7. Same as in figure 6 but for a binary of two IMBH (left panel) and an IMRI composed of an intermediate-mass black hole of mass $M_{\mathrm{BH}}=3000 M_{\odot}$ and a stellarmass one of $m_{\star}=10 M_{\odot}$ (right panel). In the latter we also include information about the initial periapsis distance $R_{\mathrm{p}}^{0}$, as well as the initial time for merger $T_{\mathrm{pl}}$.

based detector LISA. Although our estimate is based upon current technology and we possibly overestimate the Moon's seismic activity, LION performs better than ET and CE at frequencies below $30 \mathrm{~Hz}$ with detection capabilities down to $0.7 \mathrm{~Hz}$. LION also manages to maintain performance comparable to $\mathrm{CE}$ into the $\mathrm{kHz}$ regime.

\section{Science case}

The LION sensitive band, from figure 5, ranges between $0.7 \mathrm{~Hz}$ and several $\mathrm{kHz}$ with peak sensitivity at $10 \mathrm{~Hz}$. Several investigations of sources in this frequency range have been conducted [95], showing the great interest in this band, a region where the current ground based network is blind to. In general, detecting in a lower frequency range opens the possibility for observing more massive black holes and thus allows to study gravity in the strong field regime [29]. This is a key element in the testing of general relativity in more extreme limits. Many new potential sources are predicted to exist in this band, for example, type 1A supernovae, which have a gravitational wave frequency signature around $1 \mathrm{~Hz}$. As discussed in [28], their observation may resolve the open question of their origins.

What makes LION unique, however, is its ability to cover a wide regime of frequencies and characteristic strains of merging binaries which cannot be explored with any other observatory. This forms the focus for this section.

\subsection{An approximation}

We first give a few examples approximating the evolution in phase-space of the harmonics of the characteristic strain with the method of Peters and Mathews [96]. This has the advantage of allowing us to explore the evolution of the eccentricity, which is rather limited in more realistic waveforms based on computations of full general relativity. Once we have an idea of the evolution in phase-space of these systems, we will investigate in more detail the evolution of the full waveform and the computation of the signal-to-noise ratio (SNR) following the 


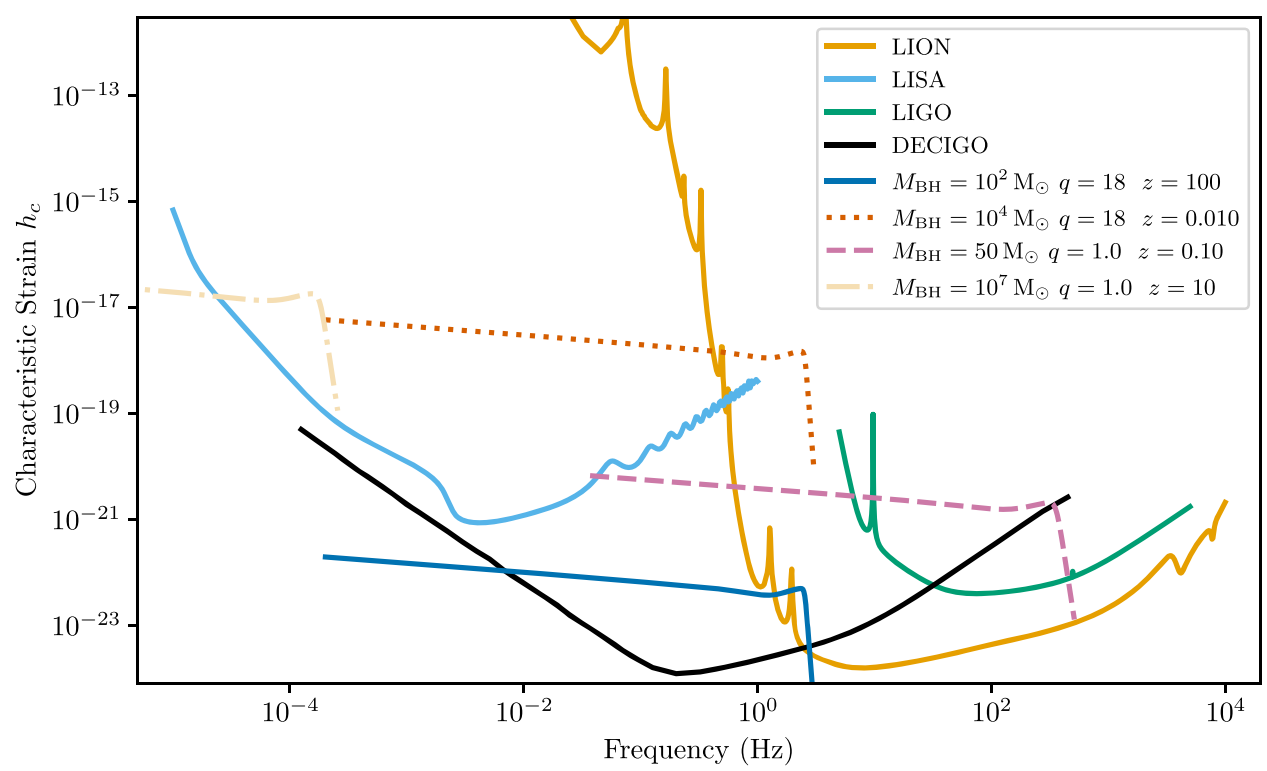

Figure 8. Evolution of four different signals with masses ranging between $10^{7} M_{\odot}$ and $50 M_{\odot}$, different mass ratios $q$ (in the legend we show the mass of the largest black hole) and the assumed redshift. We show three different observatories (LISA, LION and LIGO). The yellow, dash-dotted waveform corresponds to a system of $10^{7} M_{\odot}$ at a redshift $z=10$ with $q=1$; the orange, solid waveform to a binary of $q=100 M_{\odot}$, $z=100$ and $q=18$; the blue, dotted one to a system of $10^{4} M_{\odot}, z=10^{-2}, q=18$ and the green, dashed curve represents a binary of mass $50 M_{\odot}, z=0.1$ and $q=1$.

method of Kaiser and McWilliams [97], which we will describe in some detail. The scheme of Peters and Mathews [96] is based on an approximation of Keplerian ellipses. If we assume that the orbital parameters change slowly due to the emission of gravitational radiation, and that the gravitational waves are emitted at the integer multiples of the orbital frequency, the strain amplitude in the $n$th harmonic can be described as

$$
\begin{aligned}
h_{n}= & g(n, e) \frac{G^{2} M_{1} M_{2}}{D a c^{4}} \\
\simeq & 1.6 \times 10^{-22} g(n, e)\left(\frac{D}{1 \mathrm{Gpc}}\right)^{-1}\left(\frac{a}{10^{-2} \mathrm{pc}}\right)^{-1}\left(\frac{M_{1}}{4 \times 10^{4} M_{\odot}}\right) \\
& \times\left(\frac{M_{2}}{10 M_{\odot}}\right),
\end{aligned}
$$

where we have normalised to parameters corresponding to an intermediate-mass ratio inspiral (IMRI, see e.g. [29, 31-33, 98]), $g(n, e)$ is a function of the harmonic number $n, D$ being the distance between them, and the eccentricity of the source $e$. The full frequency evolution of the strain amplitude is discussed in section 4.2. In figure 6 we give an example of an IMRI and the inspiral of a binary of two stellar-mass black holes with masses $30 M_{\odot}$.

Another interesting example is shown in figure 7. A system of two intermediate-mass black holes (IMBH) and an IMRI which, again, are only observable by LION at these distances and 


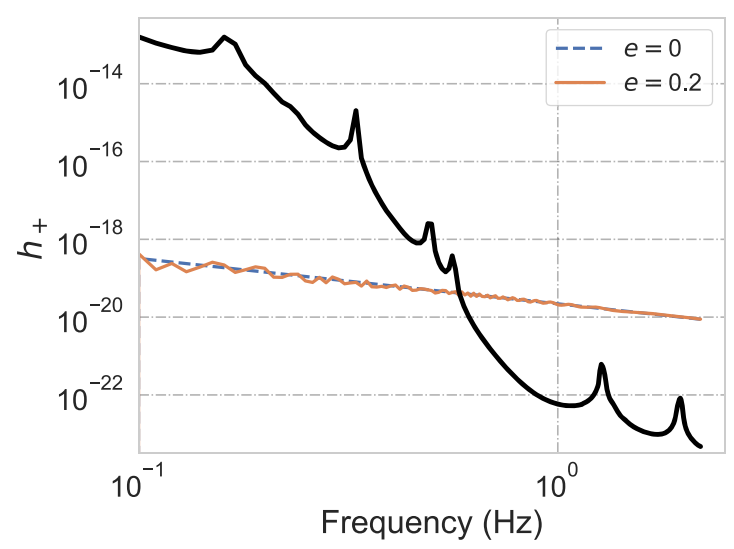

Figure 9. Evolution of the plus polarization $h_{+}$for a binary of two IMBHs of masses $M_{1}=M_{2}=10^{3} M_{\odot}$ in the LION window (solid, black line). We consider two different eccentricities, one zero and the other $e=0.2$ at the beginning of the evolution. As the systems evolve, the loss of angular momentum circularises the eccentric case, which converges toward the circular one. The binaries are assumed to be at a distance of $1 \mathrm{Gpc}$. For LION, the circular- and eccentric cases yield SNR $\sim 1855$ and 1875, respectively, while for (Advanced) LIGO the values are $\sim 3.7$ for the circular case, and 3.8 for the eccentric one.

parameters. As we can see in the left panel, LION combined with LISA would allow us to do multi-bandwidth gravitational wave astronomy (see e.g. [26]). Since we can observe a source with LISA in the inspiral phase years before the final merger, we can predict the time it is seen in the lion band with an accuracy on the order of a few seconds. This early warning would likely help to enhance the detection of the merger and ringdown with LION. Thanks to this, we would be in the position of breaking various degeneracies in the parameter extraction of the source. As before, we note that only LION is in this unique position.

As noted in [30], there is a need for a deci-Hertz gravitational wave observatory such as LION to understand the formation of compact binaries. This is something that LISA and LIGO/Virgo are not in the position of doing, as explained in [30].

\subsection{Realistic waveforms and one comparison to LIGO}

After our first exploration of the possible interesting systems for LION to observe, we now address with more realistic waveforms some examples. In particular, we employ the method of [101, 102], commonly referred to as IMRPhenomD, via the approach of [97]. The family IMRPhenomD approaches coalescing binaries by compounding a post-Newtonian approximation for the inspiral with a fully numerical relativistic solution for the merger and ringdown. This hybrid method allows us to quickly compute different waveforms. We also consider the approximation of [103], ENIGMA, which is a time domain, inspiral-merger-ringdown waveform model which allows consideration of moderately eccentric orbits, via the implementation of [104]. In particular, we display in figure 8 four sources, with one of them only in the LISA regime as a reference point.

Probably the most remarkable system is a binary of two black holes of mass ratio $q=18$ with the heaviest one having a mass of $100 M_{\odot}$ at a redshift of $z=100$ (lowermost signal). This binary is only detectable by LION and shows the relevance of the detector. 


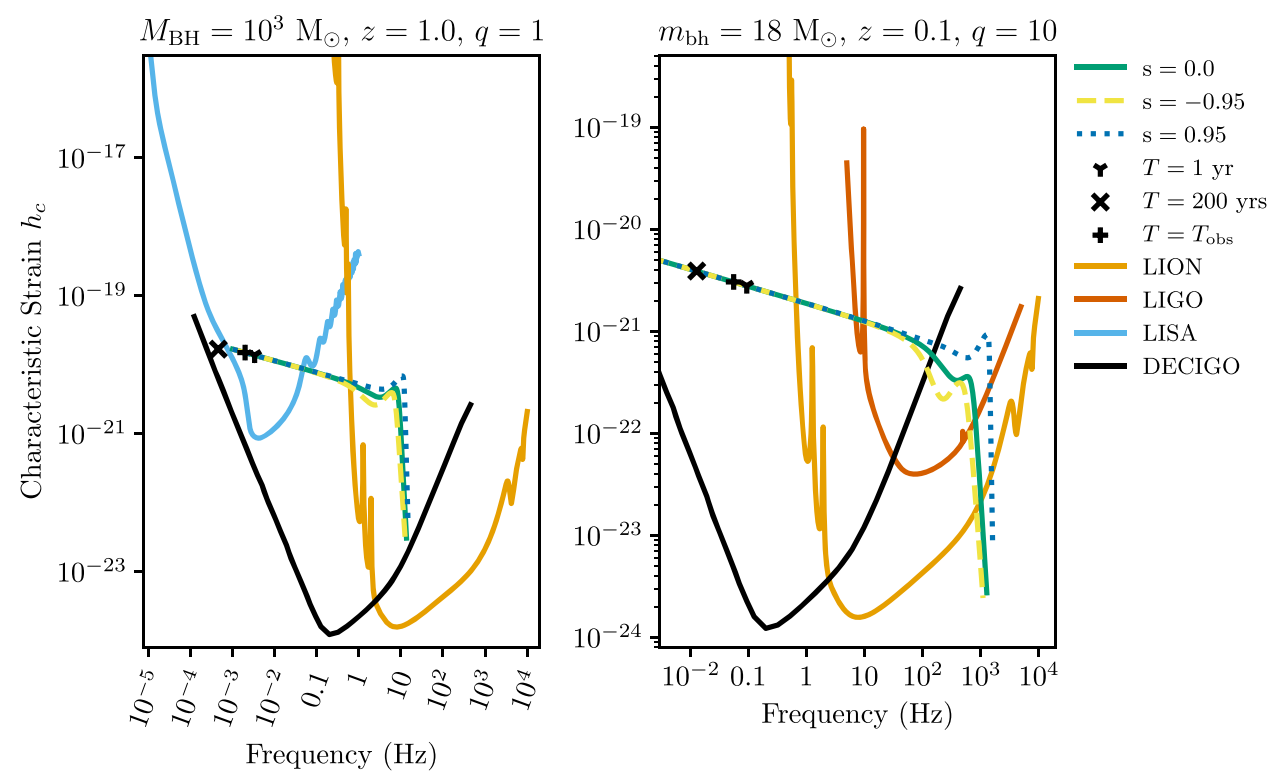

Figure 10. Left panel: a binary of two IMBHs of masses $10^{3} M_{\odot}$ at a redshift $z=1$ for different spin values (see legend on the right) at three different moments, namely 200 years before the coalescence, at the detector's (typical) observation time $T_{\mathrm{obs}}$ and 1 year before the coalescence. We display the sensitivity curve of LISA (blue, left one) and LION (orange, right one). Right panel: evolution of the waveform for a binary of two black holes with $q=18$ and the mass of the heaviest one $m_{\mathrm{bh}}=18 M_{\odot}$, at a redshift $z=0.1$. As in the left panel, we pinpoint the same moments in the evolution, different spin configurations, the sensitivity curve of LION (orange, left one) and LIGO (red, right one).

We show in figure 9 a binary of two IMBHs with equal massand different eccentricities, as approximated with ENIGMA. The case with eccentricity yields a slightlyhigher signal-to-noise ratio (SNR) than that calculated for chirping binaries.

Because IMRPhenomD features mass ratios up to 18 including spins, in figure 10 we show the role of the spin $s$ and $q$ for two different systems, a binary of two IMBHs and a lighter one, with the mass of the heaviest black hole $18 M_{\odot}$, and $q=10$. As expected, a mass ratio and positive spin value increases the characteristic strain as we approach the end of the evolution, which has an impact on the calculation of the SNR.

\subsection{Signal-to-noise ratio contour maps}

To estimate the SNR for different configurations and systems, we adopt the approach of [24] and assume that the noise is stationary, Gaussian and uncorrelated with the signal. If we envisage the waveforms as vectors in a Hilbert space [105], we can define a noise-weighted inner product as follows

$$
\left\langle h^{\prime} \mid h\right\rangle:=2 \int_{0}^{\infty} \mathrm{d} f \frac{\tilde{h^{\prime}}(f) \tilde{h}(f)^{*}+\tilde{h}^{\prime}(f)^{*} \tilde{h}(f)}{S_{n}(f)} .
$$

In the last equation we have introduced $\tilde{h}(f)$ as the Fourier transform of the time-domain waveform. Moreover, $S_{n}(f)$ is the one-sided noise power spectral density of the detector, following 


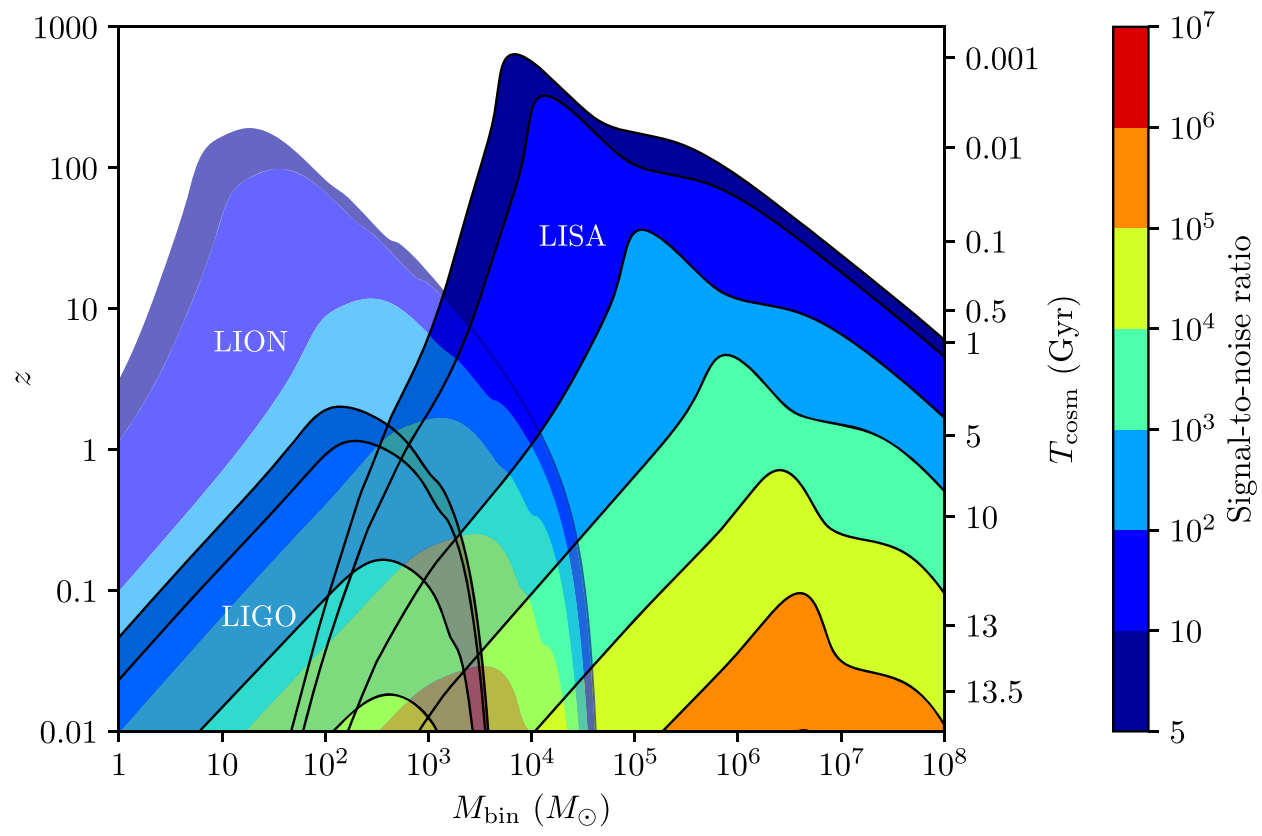

Figure 11. Contours of SNR as a function of the total mass of the binary system $M_{\text {bin }}$ in solar masses and redshift (and cosmological time, in Gyrs). We remark how LION covers a region of phase-space which is totally inaccessible to both LISA and LIGO, as shown with particular examples in figure 8 .

[106, 107], and see section 2.3 of [97] for a summary and implementation. If we adopt the above-mentioned assumptions, the optimal SNR when filtering the data against $h$ is given by

$$
\rho=\sqrt{\left\langle h_{+} \mid h_{+}\right\rangle+\left\langle h_{\times} \mid h_{\times}\right\rangle} .
$$

In figure 11 we depict the SNR $\rho$ as a function of the total mass of the binary $M_{\text {bin }}$ and redshift for LION, but also LIGO and LISA for a reference point. We note that LION is in the position of extracting information about binaries with masses in the regime necessary to understand the formation and cosmical evolution of supermassive black holes (see, for a review [25]), with SNRs values at redshift exceeding 100.

Finally, in figure 12 we show how the SNR depends on the redshift, mass ratio and spin values for LION and compare it with LIGO as a reference. We note that, contrary to figure 11, the contour map covers SNRs including values from 1.

\subsection{Angular resolution}

We note that the frequency range of LION allows us to improve the angular resolution of source positioning in the sky, by adding a lunar detector to the LVK collaboration. The angular resolution $\Delta \Omega$ is proportional to the increase of the network area $A, \Delta \Omega \propto 1 / A$ [27]. This means that an average Earth-Moon distance of $380000 \mathrm{~km}$ [108] increases the area A and improves the angular resolution by a factor of 30 compared to a purely Earth based network with maximum distance of $12000 \mathrm{~km}$.

This will not only help to enhance the sky mapping of these objects, but also opens a huge possibility to multi messenger astronomy, as we have seen, in particular in figure 11 . The 

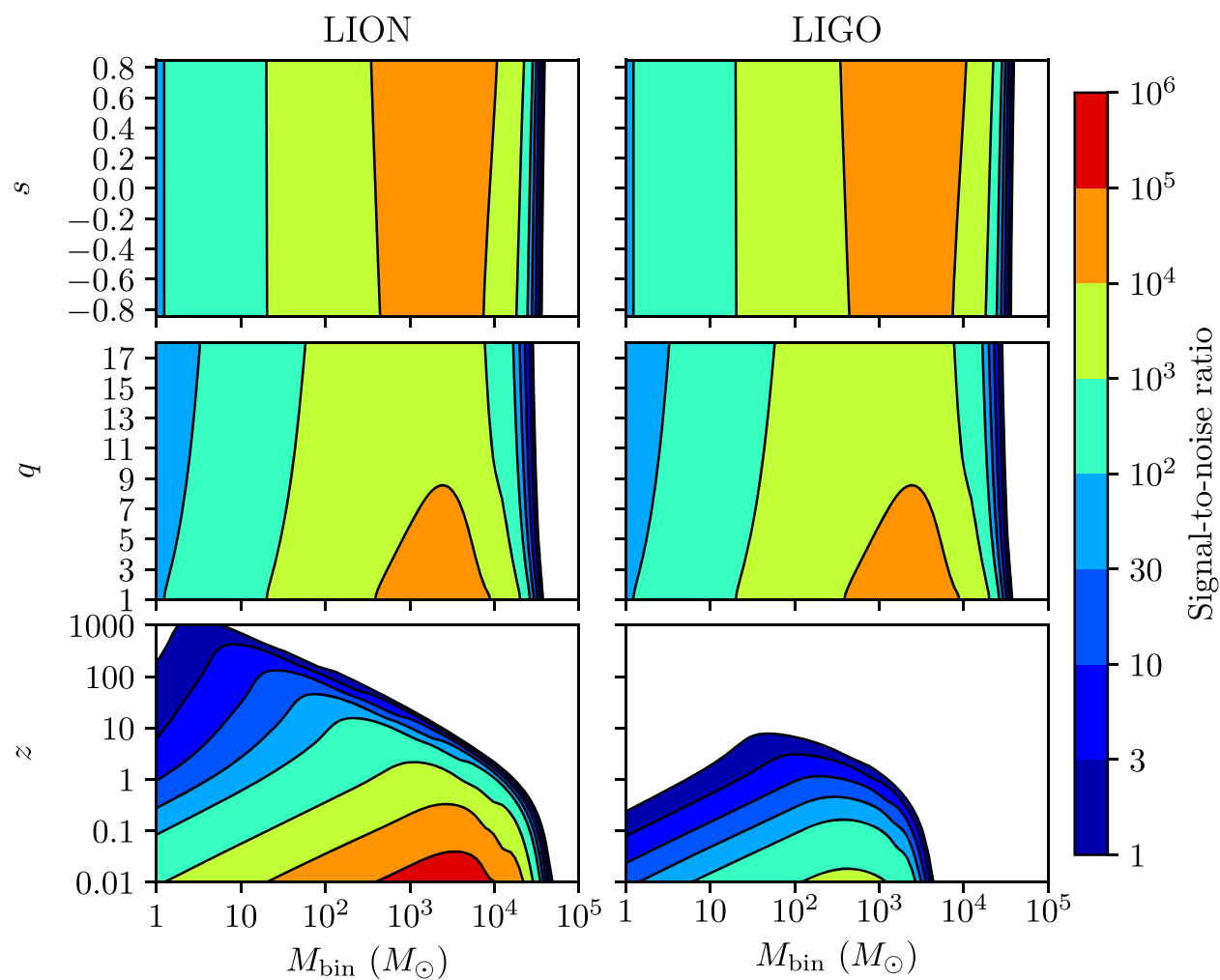

Figure 12. Mosaique of SNR contour maps to show its dependency with the redshift (bottom panels), as in figure 11), the mass ratio $q$ ranging between the values that IMRPhenomD allows for (mid panels, see text) and the spin (top panels). We show LION on the left column and LIGO on the right one.

first counterpart observation GW170817 [109], showed, how much knowledge is gained when observing the emitted gravitational waves and electromagnetic radiation from an event. With the combination of improved sky location and knowledge about the time of the merger beforehand, the electromagnetic telescopes can be pointed to the sky location in time to ensure a simultaneous observation. This will significantly increase the upcoming gravitational-wave optical transit observer array's useful observations [110].

\section{Cost and timeline}

The costs for LION can only be roughly estimated at this point. We want to stress that LION should focus on using existing gravitational wave detector technologies as well as by then existing space flight technologies. Still, we assume here a conservative budget, highly dependent on technological investigations of the next years. Based upon a study from the Center for Strategic and International Studies [111], an infrastructure on the Moon and its installation, including the launch and landers, can be approximated to cost 30 billion $€$, from which we exclude the $14 \mathrm{~B} €$ for the Luna base itself. The CE development costs are currently assumed to be about $55 \mathrm{M} €$ [6]. Assuming, that upgrading vacuum compatibility to space compatibility is a factor of 10 in costs, this leads to $0.55 \mathrm{~B} €$ for the development. Meanwhile, the total costs of LIGO are assumed to be in the order of $500 \mathrm{M} €$ [112]. Bringing 
these numbers together gives us a conservative estimate for LION of $(30-14+0.55+5)$ $\mathrm{B} €=21.55 \mathrm{~B} €$

With an initial mission proposal by 2023 and a full design study based on new data about the Moon by 2030, simultaneous development of technologies along side CE's second phase would enable us to begin space qualifying the necessary parts so that all technologies are space qualified by 2048. This would give time to build the missions in time for three launches in the middle of the decade. Assembling would be done robotically and it is hoped that this will be completed within a couple of years. The mission lifetime is expected to be 10 years, with the possibility of 5 years expansion. It is expected that in this time components will wear out and limit operation. However, if there proved to be sufficient interest, LION can continuously be repaired and upgraded, making an advanced LION detector more likely then the need of disposal. This, again, is meant to be a conservative estimate. An acceleration of the progress, to overlap with the planned LISA mission launching in 2034 is highly recommended.

\section{Conclusion}

We find that a detector built on the Moon with technology of the third generation detectors features a detection bandwidth between $0.7 \mathrm{~Hz}$ and $10 \mathrm{kHz}$ with detection noise hitting a floor of $3 \times 10^{-24} \mathrm{~m} \mathrm{~Hz}^{-1 / 2}$ at $10 \mathrm{~Hz}$. This is a conservative estimate which is limited by the thermal noise and residual seismic activity of the Moon. Since the actual seismic activity of the Moon is still uncertain and we only use an upper estimate of activity, a thorough test campaign is likely to improve the performance prediction. Nevertheless, LION would make an excellent complement to the existing network, with significant overlap of required technology development.

The unique detection band presents an opportunity to push the capabilities of gravitational wave astronomy and our understanding of physics. The ability to measure binaries of seed black holes to a redshifts of 100 and higher provides a unique glance at the cauldron where stars and galaxies were formed and would shed light on the question on how massive black holes assemble their mass and grow over cosmic timescales (see e.g. [25], for a review). We also find that many of the sources present in the LISA band can be picked up in the LION band to observe final merger achieving the long held dream of multi messenger astronomy. Doing so will break many of the degeneracies of the parameter space so that we can better understand their formation channels [30]. In this regard, it can be envisaged as a true deci-Hertz bridge between space borne observatories and ground based ones. Moreover, having a much wider detection array at low frequencies is imperative for the coordination between electromagnetic and gravitational observations needed to glimpse the final stages of an inspiraling system with an optical telescope. Thus, LION will reach its full potential when being operated together with the third generation detectors on the Earth and in space.

The Moon is perhaps one of the best available environments to operate a gravitational wave detector in our Solar System. While we show in this paper that current or currently designed technology enables the low frequency detector, its installation on the lunar surface requires significant technological development. However, these lie not too far beyond the horizon of humanities progress. We believe that, once humanity starts exploiting the Moon for science and resources, a gravitational wave observatory is an excellent candidate for the first large scale infrastructure experiment on the lunar surface. 


\section{Author contributions}

The initial idea of LION was developed by the authors Bischof, Carter, Hartig and Wilken at a lecture week in 2018, where Amaro-Seoane was supervising, run by the International Max Planck Research School for Gravitational Waves (IMPRS-GW). Bischof, Carter, Hartig and Wilken followed up the lecture week with a deeper exploration of the required mission parameters, noise curves and technical discussion of the paper. The work on sources in the detection band was performed by Pau Amaro Seoane.

\section{Acknowledgments}

This paper builds on a student project from an lecture week by the International Max-Planck Research School for Gravitational Waves (IMPRS-GW) in 2018. Therefore we would like to thank the IMPRS-GW for organisation and Karsten Danzmann for providing the space necessary for the development of this research. We thank Stefan Danilishin and James Lough for the discussion of the technical and the environmental challenges. We are also thankful to Christoph Vorndamme for discussion on the initial noise budgets. We thank Frank Ohme for his comments and suggestions, as well as Andrew Kaiser for comments and for making his approach publicly available. Finally, we would like to thank Harald Lück and Jens Reiche for useful feedback on the manuscript. We also acknowledge that the work of the authors Bischof, Carter, Hartig and Wilken was supported by the IMPRS-GW grant and the funding by the Deutsche Forschungsgemeinschaft in the Collaborative Research Center SFB1128 Relativistic Geodesy and Gravimetry with Quantum Sensors (geo-Q) at Leibniz Universität Hannover. Bischof, Carter, Hartig and Wilken gratefully acknowledge support by the Deutsches Zentrum für Luft-und Raumfahrt (DLR) with funding from the Bundesministerium für Wirtschaft und Technologie (FKZ 50OQ1801). Amaro-Seoane acknowledges support from the Ramón y Cajal Program of the Ministry of Economy, Industry and Competitiveness of Spain, as well as the financial support of Programa Estatal de Generación de Conocimiento (reference PGC2018096663-B-C43) (MCIU/FEDER), and the COST Action GWverse CA16104. His work was supported by the National Key R \& D Program of China (2016YFA0400702) and the National Science Foundation of China (11721303).

\section{Data availability statement}

The data that support the findings of this study are available upon reasonable request from the authors.

\section{ORCID iDs}

Pau Amaro-Seoane (D) https://orcid.org/0000-0003-3993-3249

Lea Bischof (ID https://orcid.org/0000-0003-1576-7274

Jonathan J. Carter (D) https://orcid.org/0000-0001-8845-0900

Marie-Sophie Hartig (D) https://orcid.org/0000-0002-1078-3497

Dennis Wilken (D) https://orcid.org/0000-0002-7290-9411 


\section{References}

[1] LIGO Scientific Collaboration and Advanced LIGO 2015 Class. Quantum Grav. 32074001

[2] Caron B et al 1997 The Virgo interferometer Class. Quantum Grav. 141461

[3] Aso Y, Michimura Y, Somiya K, Ando M, Miyakawa O, Sekiguchi T, Tatsumi D and Yamamoto H 2013 Interferometer design of the KAGRA gravitational wave detector Phys. Rev. D 88043007

[4] Punturo M et al 2010 The Einstein telescope: a third-generation gravitational wave observatory Class. Quantum Grav. 27194002

[5] Dwyer S, Sigg D, Ballmer S W, Barsotti L, Mavalvala N and Evans M 2015 Gravitational wave detector with cosmological reach Phys. Rev. D 91082001

[6] Reitze D et al 2019 Cosmic explorer: the US Contribution to gravitational-wave astronomy beyond LIGO (arXiv:1907.04833)

[7] Beker M G, van den Brand J F, Hennes E and Rabeling D S 2012 Newtonian noise and ambient ground motion for gravitational wave detectors J. Phys.: Conf. Ser. 363012004

[8] Amaro-Seoane P et al 2017 Laser interferometer space antenna (arXiv:1702.00786v3)

[9] Essick R, Vitale S and Evans M 2017 Frequency-dependent responses in third generation gravitational-wave detectors Phys. Rev. D 96084004

[10] Schilling R 1997 Angular and frequency response of LISA Class. Quantum Grav. 14 1513-9

[11] Manchester R N, Hobbs G B, Teoh A and Hobbs M 2005 The Australia telescope National Facility Pulsar Catalogue Astron. J. 129 1993-2006

[12] Kawamura S et al 2006 The Japanese space gravitational wave antenna-DECIGO Class. Quantum Grav. $23 \mathrm{~S} 125$

[13] Kawamura S et al 2011 The Japanese space gravitational wave antenna: DECIGO Class. Quantum Grav. 28094011

[14] Sato S et al 2017 The status of DECIGO J. Phys.: Conf. Ser. 840012010

[15] Gregory H et al 2003 Laser interferometry for the Big Bang observer Class. Quantum Grav. 23 7361

[16] LaFave N and Wilson T 1993 Lunar LIGO: A New Concept in Gravitational Wave Astronomy vol 24 (Lunar and Planetary Institute) pp 841-2

[17] Wilson T and LaFave N 1994 Lunar LIGO and gravitational wave astronomy on the Moon Lunar and Planetary Science Conf. vol 25 pp 1499-500

[18] Paik H J and Venkateswara K Y 2009 Gravitational wave detection on the Moon and the moons of Mars Adv. Space Res. 43 167-70

[19] Coughlin M and Harms J 2014 Constraining the gravitational-wave energy density of the Universe in the range $0.1 \mathrm{~Hz}$ to $1 \mathrm{~Hz}$ using the Apollo seismic array Phys. Rev. D 90102001

[20] Harms J et al 2021 Lunar gravitational-wave antenna Astrophys. J. 9101

[21] Jani K and Loeb A 2020 Gravitational-wave lunar observatory for cosmology (arXiv:200708550)

[22] Friedrich D, Nakano M, Kawamura H, Yamanaka Y, Hirobayashi S and Kawamura S 2014 Juggled interferometer for the detection of gravitational waves around $0.1-10 \mathrm{~Hz}$ Class. Quantum Grav. 31245006

[23] Latham G V et al 1970 Passive seismic experiment Science 167 455-7

[24] Maggiore M 2008 Gravitational Waves: Volume 1: Theory and Experiments. Gravitational Waves (Oxford: Oxford University Press) Available from: https://books.google.de/books?id\&tnqx3d; AqVpQgAACAAJ

[25] Colpi M and Dotti M 2011 Massive binary black holes in the cosmic landscape Adv. Sci. Lett. 4 $181-203$

[26] Amaro-Seoane P and Santamaría L 2010 Detection of imbhs with ground-based gravitational wave observatories: a biography of a binary of black holes, from birth to death Astrophys. J. $\mathbf{7 2 2}$ 1197-206

[27] Wen L and Chen Y 2010 Geometrical expression for the angular resolution of a network of gravitational-wave detectors Phys. Rev. D 81082001

[28] Mandel I, Sesana A and Vecchio A 2018 The astrophysical science case for a decihertz gravitational-wave detector Class. Quantum Grav. 35054004

[29] Amaro-Seoane P 2018 Detecting intermediate-mass ratio inspirals from the ground and space Phys. Rev. D 98063018

[30] Chen X and Amaro-Seoane P 2017 Revealing the formation of stellar-mass black hole binaries: the need for deci-Hertz gravitational-wave observatories Astrophys. J. 842 L2 
[31] Amaro-Seoane P 2018 Relativistic dynamics and extreme mass ratio inspirals Living Rev. Relativ. 214

[32] Amaro-Seoane P 2020 The gravitational capture of compact objects by massive black holes (arXiv:2011.03059)

[33] Amaro-Seoane P et al 2007 Intermediate and extreme mass-ratio inspirals. Astrophysics, science applications and detection using LISA Class. Quantum Grav. 24113

[34] Mitrofanov I, Dolgopolov V, Khartov V, Lukjanchikov A, Tret'yakov V and Zelenyi L 2014 'Luna-Glob' and 'Luna-Resurs': science goals, payload and status EGU General Assembly Conf. Abstracts $16 \mathrm{p} 1$

[35] Sibeck D et al 2011 ARTEMIS science objectives The ARTEMIS Mission (New York: Springer) pp 27-59

[36] Xiao L 2014 China's touch on the Moon Nat. Geosci. 7391

[37] Haruyama J, Sawai S, Mizuno T, Yoshimitsu T, Fukuda S and Nakatani I 2012 Exploration of lunar holes, possible skylights of underlying lava tubes, by smart lander for investigating moon (slim) Trans. Jpn. Soc. Aeronaut. Space Sci. 10 Pk_7-Pk_10

[38] Adhikari R 2020 Gravitational wave interferometer noise calculator https://git.ligo.org/gwinc/ pygwinc

[39] Hayne P O, Hendrix A, Sefton-Nash E, Siegler M A, Lucey P G, Retherford K D, Williams J-P, Greenhagen B T and Paige D A 2015 Evidence for exposed water ice in the Moon's south polar regions from lunar reconnaissance orbiter ultraviolet albedo and temperature measurements Icarus 255 58-69 Available from: http://sciencedirect.com/science/article/pii/ S0019103515001335

[40] Ingersoll A P, Svitek T and Murray B C 1992 Stability of polar frosts in spherical bowl-shaped craters on the Moon, Mercury, and Mars Icarus 100 40-7 Available from: http://sciencedirect. com/science/article/pii/001910359290016Z

[41] Williams J-P, Paige D A, Greenhagen B T and Sefton-Nash E 2017 The global surface temperatures of the moon as measured by the diviner lunar radiometer experiment Icarus 283 300-25 Available from: http://sciencedirect.com/science/article/pii/S0019103516304869

[42] Sharpe B L and Schrunk D G 2003 Malapert mountain: gateway to the moon Adv. Space Res. 31 2467-72 The Moon: Science, Exploration and Utilisation. Available from: http://sciencedirect. com/science/article/pii/S0273117703005350

[43] Williams D R (ed) 2020 Moon Fact Sheet (Washington, DC: NASA)

[44] Danzmann K (The LISA study team) 1996 LISA: laser interferometer space antenna for gravitational wave measurements Class. Quantum Grav. 13 A247-50

[45] McNamara P, Vitale S and Danzmann K 2008 Class. Quantum Grav. 25114034

[46] Latham G V et al 1970 Apollo 11 passive seismic experiment Geochim. Cosmochim. Acta 12309

[47] Nakamura Y 1980 Shallow moonquakes: how they compare with earth-quakes Lunar and Planetary Science Conf. Proc. vol 3 1847-53

[48] Lognonné P, Feuvre M L, Johnson C L and Weber R C 2009 Moon meteoritic seismic hum: steady state prediction J. Geophys. Res.: Planets 12114

[49] Banerdt W B et al 2007 Using the Moon as a low-noise seismic detector for strange quark nuggets Nucl. Phys. B 166 203-8 Proc. of the Third Int. Conf. on Particle and Fundamental Physics in Space. Available from: http://sciencedirect.com/science/article/pii/S0920563206010188

[50] Watters T R, Weber R C, Collins G C, Howley I J, Schmerr N C and Johnson C L 2019 Shallow seismic activity and young thrust faults on the Moon Nat. Geosci. 12 411-7

[51] Beker M G et al 2011 Improving the sensitivity of future GW observatories in the 1-10 Hz band: Newtonian and seismic noise Gen. Relativ. Gravit. 43 623-56

[52] Matichard F et al 2015 Advanced LIGO two-stage twelve-axis vibration isolation and positioning platform. Part 2: experimental investigation and tests results Precis. Eng. 40 287-97

[53] Lantz B, Schofield R, O'Reilly B, Clark D E and DeBra D 2009 Review: requirements for a ground rotation sensor to improve advanced LIGO Bull. Seismol. Soc. Am. 99 980-9

[54] Arai K and Collaboration T 2002 Sensing and controls for power-recycling of TAMA300 Class. Quantum Grav. 19 1843-8

[55] Carter J J, Cooper S J, Thrift E, Briggs J, Warner J, Ross M P and Mow-Lowry C M 2020 Particle swarming of sensor correction filters Class. Quantum Grav. 37205009

[56] Dacey J 2019 The new race to the Moon Phys. World 32 10-1 
[57] Mitrofanov I G, Zelenyi L M, Tret'yakov V I and Dolgopolov V P 2011 Science program of lunar landers of 'Luna-Glob' and 'Luna-Resource' missions Lunar and Planetary Science Conf. p 1798

[58] Cumming A V et al 2012 Design and development of the advanced LIGO monolithic fused silica suspension Class. Quantum Grav. 29035003

[59] Aston S M et al 2012 Update on quadruple suspension design for Advanced LIGO Class. Quantum Grav. 29235004

[60] Saulson P R 1994 Fundamentals of Interferometric Gravitational Wave Detectors (Singapore: World Scientific)

[61] Schroeter A et al 2007 On the mechanical quality factors of cryogenic test masses from fused silica and crystalline quartz (arXiv:0709.4359)

[62] Penn S D et al 2003 Mechanical loss in tantala/silica dielectric mirror coatings Class. Quantum Grav. 202917

[63] Hirose E et al 2014 Update on the development of cryogenic sapphire mirrors and their seismic attenuation system for KAGRA Class. Quantum Grav. 31224004

[64] Nawrodt R et al 2008 High mechanical $Q$-factor measurements on silicon bulk samples $J$. Phys.: Conf. Ser. 122012008

[65] Cumming A V et al 2013 Silicon mirror suspensions for gravitational wave detectors Class. Quantum Grav. 31025017

[66] Vajente $\mathrm{G}$ et al 2018 Effect of elevated substrate temperature deposition on the mechanical losses in tantala thin film coatings Class. Quantum Grav. 35075001

[67] Bunkowski A, Burmeister O, Friedrich D, Danzmann K and Schnabel R 2006 High reflectivity grating waveguide coatings for $1064 \mathrm{~nm}$ Class. Quantum Grav. 23 7297-303

[68] Principe M 2015 Reflective coating optimization for interferometric detectors of gravitational waves Opt. Express 23 10938-56

[69] Lough $\mathrm{J}$ et al 2021 First demonstration of $6 \mathrm{~dB}$ quantum noise reduction in a kilometer scale gravitational wave observatory Phys. Rev. Lett. 126041102

[70] Acernese F et al 2019 Increasing the astrophysical reach of the advanced Virgo detector via the application of squeezed vacuum states of light Phys. Rev. Lett. 123231108

[71] Tse M et al 2019 Quantum-enhanced advanced LIGO detectors in the era of gravitational-wave astronomy Phys. Rev. Lett. 123231107

[72] Zhao Y et al 2020 Frequency-dependent squeezed vacuum source for broadband quantum noise reduction in advanced gravitational-wave detectors Phys. Rev. Lett. 124171101

[73] Ma Y, Miao H, Pang B H, Evans M, Zhao C, Harms J, Schnabel R and Chen Y 2017 Proposal for gravitational-wave detection beyond the standard quantum limit through EPR entanglement Nat. Phys. 13 776-80

[74] Staley A et al 2014 Achieving resonance in the Advanced LIGO gravitational-wave interferometer Class. Quantum Grav. 31245010

[75] Mullavey A J, Slagmolen B J J, Miller J, Evans M, Fritschel P, Sigg D, Waldman S J, Shaddock D A and McClelland D E 2012 Arm-length stabilisation for interferometric gravitationalwave detectors using frequency-doubled auxiliary lasers Opt. Express 20 81-9 Available from: http://opticsexpress.org/abstract.cfm?URI=oe-20-1-81

[76] Biscans S et al 2018 Control strategy to limit duty cycle impact of earthquakes on the LIGO gravitational-wave detectors Class. Quantum Grav. 35055004

[77] Beckman M 2007 Mission design for the lunar reconnaissance orbiter 29th Annual AAS Guidance and Control Conf. p 1

[78] Berg O E, Wolf H and Rhee J 1976 Lunar soil movement registerd by the Apollo 17 cosmic dust experiment Interplanetary Dust and Zodiacal Light (New York: Springer) pp 233-7

[79] Colwell J E, Batiste S, Horányi M, Robertson S and Sture S 2007 Lunar surface: dust dynamics and regolith mechanics Rev. Geophys. 452

[80] Corbitt T and Mavalvala N 2004 Review: quantum noise in gravitational-wave interferometers $J$. Opt. B: Quantum Semiclass. Opt. 6 S675

[81] Brooks A F et al 2021 Point absorbers in advanced LIGO (arXiv:2101.05828)

[82] Calle C I et al 2006 Electrodynamic dust shield for surface exploration activities on the Moon and Mars 57th Int. Astronautical Congress p A5-2 https://arc.aiaa.org/doi/abs/10.2514/6.IAC-06A5.2.07

[83] Mazumder M K, Sharma R, Biris A S, Zhang J, Calle C and Zahn M 2007 Self-cleaning transparent dust shields for protecting solar panels and other devices Part. Sci. Technol. 25 5-20 
[84] Calle C I, Buhler C R, McFall J L and Snyder S J 2009 Particle removal by electrostatic and dielectrophoretic forces for dust control during lunar exploration missions J. Electrost. 67 89-92 11th Int. Conf. on Electrostatics. Available from: https://sciencedirect.com/science/article/pii/ S030438860900103X

[85] Calle C I, Buhler C R, Johansen M R, Hogue M D and Snyder S J 2011 Active dust control and mitigation technology for lunar and Martian exploration Acta Astronaut. 69 1082-8 Available from: https://sciencedirect.com/science/article/pii/S0094576511001883

[86] Kawamoto H and Miwa T 2011 Mitigation of lunar dust adhered to mechanical parts of equipment used for lunar exploration J. Electrost. 69 365-9 Available from: https://sciencedirect. com/science/article/pii/S0304388611000684

[87] Kawamoto H, Uchiyama M, Cooper B L and McKay D S 2011 Mitigation of lunar dust on solar panels and optical elements utilizing electrostatic traveling-wave J. Electrost. 69 370-9 Available from: http://sciencedirect.com/science/article/pii/S0304388611000696

[88] Kawamoto H and Hashime S 2018 Practical performance of an electrostatic cleaning system for removal of lunar dust from optical elements utilizing electrostatic traveling wave J. Electrost. 94 38-43 Available from: https://sciencedirect.com/science/article/pii/S0304388618300524

[89] Sun Q, Yang N, Cai X and Hu G 2012 Mechanism of dust removal by a standing wave electric curtain Sci. China Phys. Mech. Astron. 55 1018-25

[90] Afshar-Mohajer N, Wu C-Y, Moore R and Sorloaica-Hickman N 2014 Design of an electrostatic lunar dust repeller for mitigating dust deposition and evaluation of its removal efficiency $J$. Aerosol Sci. 69 21-31

[91] Dove A, Devaud G, Wang X, Crowder M, Lawitzke A and Haley C 2011 Mitigation of lunar dust adhesion by surface modification Planet. Space Sci. 59 1784-90

[92] Afshar-Mohajer N, Wu C-Y, Curtis J S and Gaier J R 2015 Review of dust transport and mitigation technologies in lunar and Martian atmospheres Adv. Space Res. 56 1222-41

[93] Chavers D G 2016 NASA lander technologies project status AIAA Space 2016 (Aerospace Research Central) p 5221

[94] Boroson D M et al 2014 Overview and results of the lunar laser communication demonstration Free-Space Laser Communication and Atmospheric Propagation XXVI vol 8971 https://doi.org/10.1117/12.2045508

[95] Gair J R, Mandel I, Miller M C and Volonteri M 2011 Exploring intermediate and massive blackhole binaries with the Einstein telescope Gen. Relativ. Gravit. $43485-518$

[96] Peters P C and Mathews J 1963 Gravitational radiation from point masses in a keplerian orbit Phys. Rev. 131 435-40

[97] Kaiser A R and McWilliams S T 2020 Sensitivity of present and future black-hole binary observations across the gravitational wave spectrum (arXiv:2010.02135)

[98] Konstantinidis S, Amaro-Seoane P and Kokkotas K D 2013 Investigating the retention of intermediate-mass black holes in star clusters using N-body simulations Astron. Astrophys. 557 A135

[99] Peters P C 1964 Gravitational radiation and the motion of two point masses Phys. Rev. 136 1224-32

[100] Sathyaprakash B et al 2012 Scientific objectives of Einstein telescope Class. Quantum Grav. 29 124013

[101] Husa S, Khan S, Hannam M, Pürrer M, Ohme F, Forteza X J and Bohé A 2016 Frequency-domain gravitational waves from nonprecessing black-hole binaries. I. New numerical waveforms and anatomy of the signal Phys. Rev. D 93044006

[102] Khan S, Husa S, Hannam M, Ohme F, Pürrer M, Forteza X J and Bohé A 2016 Frequency-domain gravitational waves from nonprecessing black-hole binaries. II. A phenomenological model for the advanced detector era Phys. Rev. D 93044007

[103] Huerta E A et al 2018 Eccentric, nonspinning, inspiral, Gaussian-process merger approximant for the detection and characterization of eccentric binary black hole mergers Phys. Rev. D 97 024031

[104] Nitz A et al 2020 Gwastro/pycbc: PyCBC Release v1.15.4

[105] Helstrom C W 1968 Statistical Theory of Signal Detection (Oxford: Pergamon)

[106] Thorne K S, Hawking S W and Israel W 1987 Gravitational Radiation (Cambridge: Cambridge University Press)

[107] Finn L S 1992 Detection, measurement, and gravitational radiation Phys. Rev. D 46 5236-49

[108] Murphy T W 2013 Lunar laser ranging: the millimeter challenge Rep. Prog. Phys. 76076901 
[109] Abbott B P et al 2017 GW170817: observation of gravitational waves from a binary neutron star inspiral Phys. Rev. Lett. 119161101

[110] Dyer M J et al 2020 The gravitational-wave optical transient observer (GOTO) (arXiv:2012.02685v1)

[111] Weppler J, Sabathier V and Bander A 2009 Costs of an international lunar base Center for Strategic and International Studies (CSIS) Available from: https://csis-website-prod.s3.amazonaws.com/ s3fs-public/legacy_files/files/publication/090923_weppler_lunar_base_costs.pdf

[112] Castelvecchi D 2015 Hunt for gravitational waves to resume after massive upgrade Nature $\mathbf{5 2 5}$ $301-2$ 